\title{
miRNA expression proftle of bearch Paper miRNA expression profile of bone marrow resident cells from children with neuroblastoma is not significantly different from that of healthy children
}

\author{
Sara Stigliani ${ }^{1, *}$, Fabio Morandi2,5,*, Luca Persico ${ }^{3}$, Corrado Lagazio ${ }^{3}$, Giovanni \\ Erminio ${ }^{4}$, Paola Scaruffi ${ }^{1}$ and Maria Valeria Corrias ${ }^{2}$ \\ ${ }^{1}$ Physiopathology of Human Reproduction, Ospedale Policlinico San Martino, Genoa, Italy \\ ${ }^{2}$ Experimental Therapy in Oncology, IRCCS Istituto Giannina Gaslini, Genoa, Italy \\ ${ }^{3}$ Department of Economy, University of Genoa, Genoa, Italy \\ ${ }^{4}$ Epidemiology, Biostatistics and Committees, IRCCS Istituto Giannina Gaslini, Genoa, Italy \\ ${ }^{5}$ Present address: Stem Cell Laboratory and Cell Therapy Center, IRCCS Istituto Giannina Gaslini, Genoa, Italy \\ *These authors contributed equally to this work \\ Correspondence to: Maria Valeria Corrias, email: mariavaleriacorrias@gaslini.org \\ Keywords: miRNA; neuroblastoma; bone marrow; mitochondria; gene expression \\ Received: August 29, $2017 \quad$ Accepted: March 02, $2018 \quad$ Published: April 10, 2018 \\ Copyright: Stigliani et al. This is an open-access article distributed under the terms of the Creative Commons Attribution License \\ 3.0 (CC BY 3.0), which permits unrestricted use, distribution, and reproduction in any medium, provided the original author and \\ source are credited.
}

\section{ABSTRACT}

The miRNA expression profiles of bone marrow resident cells from children with neuroblastoma were compared to that of healthy children. No significant difference was found between localized and metastatic neuroblastoma, or between children with neuroblastoma and healthy children. By considering the fold change we identified six miRNAs over-expressed by more than 150 fold in neuroblastoma. Validation confirmed miR-221 over-expression in BM resident cells from children with neuroblastoma, regardless of localized or metastatic disease.

MiR-221 over-expression was unlikely derived from neuroblastoma primary tumors or from bone marrow-infiltrating metastatic cells, since neuroblastoma cells expressed lower or similar amount of miR-221 than BM cells, respectively.

To get insight on the genes potentially regulated by miR-221 we merged the list of miR-221 potential targets with the genes under-expressed by BM resident cells from children with neuroblastoma, as compared with healthy children. In silico analysis demonstrated that none of the miR-221 target genes belonged to heme biosynthetic processes found altered in children with neuroblastoma, whereas two genes associated with mitochondria. However, the encoded proteins were not under-expressed in children with neuroblastoma, making unlikely that altered erythrocyte maturation in children with neuroblastoma was mediated by miR-221.

In conclusion, miRNA expression profiles of BM resident cells from children with localized and metastatic neuroblastoma were similar to that of BM resident cells from healthy children. Moreover, miRNAs expressed by neuroblastoma primary tumors or by BM-infiltrating NB cells do not appear to be involved in mediating the functional defect of erythrocyte maturation recently observed in children with neuroblastoma. 


\section{INTRODUCTION}

MiRNAs are 20-22 nucleotide-long non coding RNAs that, following binding to complementary sequences in the 3' UTR of an mRNA, repress translation or induce mRNA degradation. Therefore, miRNAs are considered major regulators of gene expression in both normal and neoplastic cells [1]. Since they can be found in plasma, either free or inside tumor-derived vesicles that can fuse to cells of different lineages, miRNAs can regulate gene expression also at distance [2-4].

Neuroblastoma (NB) is a pediatric tumor with a heterogeneous clinical behavior at diagnosis, varying from localized disease with good prognosis to metastatic disease with poor outcome [5]. Metastatic disease mainly involves the bone marrow (BM), and presence of BM-infiltrating NB cells is the main negative prognostic factor [6]. The role of miRNAs in regulating NB pathogenesis and invasiveness has been deeply investigated in human NB primary tumors (see $[7,8]$ for review) and NB experimental models $[9,10]$. Prognostic miRNA expression profiles of human NB primary tumors have been defined [11-13], and public datasets of miRNA expression levels in specific subsets of human NB primary tumors are available (GSE86889, GSE21713, GSE16444) [14-16].

Recently, we showed that in children with NB erythrocyte maturation was selectively impaired at the ortho-chromic stage, regardless of the presence of metastatic cells in the BM [17]. Among the genes significantly under-expressed by BM resident cells from children with NB, as compared with healthy children, we identified a set involved in heme and porphyrin biosynthesis in the mitochondria [17]. Given the pivotal role of miRNAs in regulating gene expression, here we investigated whether the decreased expression of these genes was regulated by specific miRNA(s) over-expressed by $\mathrm{BM}$ resident cells of children with $\mathrm{NB}$, as compared with healthy children. No information or public datasets of miRNA expression profiles of BM resident cells from children with NB and from healthy children were in fact available to test this hypothesis.

\section{RESULTS}

\section{miRNA expression profiles of $\mathrm{BM}$ resident cells from children with NB and from healthy children}

First, twelve BM resident cell samples from healthy children, twelve from children with localized NB and twelve from children with metastatic NB were identified in our RNA bio bank (Table 1). This cohort reflected the differences between localized and metastatic NB [5, 6]; in fact, children with localized NB were younger than those with metastatic disease, and they did not present $M Y C N$ amplification in the primary tumors, and were all alive at follow-up.
Then, the expression of 671 unique miRNAs was evaluated by high-throughput RT-qPCR [18] in four samples randomly selected from each group. Despite the clinical, demographic and genetic disparities between children with localized and metastatic NB, the miRNA expression levels in BM resident cells did not show significant differences (MiQe for qPCR in Supplementary Table 1 and raw data in Supplementary Table 2). This finding was in accordance with the absence of significant differences in gene expression profiles of BM resident cells from children with localized and metastatic NB [19].

Thus, the miRNA expression profiles of all children with NB were compared with those of healthy children. Also in this case no significant (Bonferroni's adjusted $p$ value) difference was found. Therefore, to identify miRNAs potentially over-expressed in BM resident cells from children with NB, as compared with healthy children, we ranked miRNAs according to the increased fold change (Table 2). To select miRNAs whose over-expression could be biologically relevant in regulating gene expression we considered only the six-top-ranked miRNAs with a fold increase over 150 fold (0.015 in Table 2). The expression of the six top-ranked miRNAs was then evaluated by specific RT-qPCR in all the samples. As shown in Figure 1, miR-29b, miR-202 and miR-875-5p were found expressed at similar levels in children with localized or metastatic NB and healthy children. MiR-17 was over-expressed in children with localized NB and miR-137 was significantly over-expressed in children with metastatic NB (Figure 1). Thus, only miR-221 was significantly over-expressed by BM resident cells from children with NB regardless of localized or metastatic disease (Figure 1).

\section{Expression of miR-17, miR-137 and miR-221 by NB primary tumor cells and BM-infiltrating NB cells}

To check whether the increased expression of miR17, miR-137 and miR-221 in BM resident cells from children with NB, as compared with healthy children, could be ascribed to their release from NB primary tumor cells and/or BM-infiltrating metastatic NB cells [20], the mean expression levels in all these tissues were compared (MiQe for qPCR in Supplementary Table 1, raw data in Supplementary Table 3 and 4, respectively).

As shown in Figure 2, miR-17 and miR-221 were expressed at the same level by BM resident cells from healthy children, BM-infiltrating NB cells and NB primary tumor cells $(p=n s)$, making unlikely a transfer from NB cells. Conversely, miR-137 was significantly more expressed by BM-infiltrating NB cells and NB primary tumor cells, as compared with BM resident cells from healthy children (Figure 2, $p=0.0036$ and $p=0.0053$, respectively), suggesting that miR-137 over-expression only in BM resident cells from children with metastatic NB may be dependent on its release by BM-infiltrating NB cells. 
Table 1: Main features of children with NB and healthy children

\begin{tabular}{|c|c|c|c|c|c|c|}
\hline & Localized NB & $\%$ & Metastatic NB & $\%$ & Healthy children & $\%$ \\
\hline$N$ & 12 & & 12 & & 12 & \\
\hline Stage & L1/L2 & 100 & M & 100 & NA & \\
\hline \multicolumn{7}{|l|}{ Sex } \\
\hline $\mathrm{F}$ & 6 & 50 & 4 & 33.3 & 6 & 50 \\
\hline $\mathrm{M}$ & 6 & 50 & 8 & 66.7 & 6 & 50 \\
\hline \multicolumn{7}{|l|}{ Age } \\
\hline Median (range) & $2(0.3-6.9)$ & & $5.6(1.0-12.6)$ & & $11.2(4.0-15.0)$ & \\
\hline \multicolumn{7}{|l|}{ MYCN } \\
\hline Single copy & 12 & 100 & 7 & 58.3 & & \\
\hline Amplified & 0 & - & 5 & 41.7 & & \\
\hline \multicolumn{7}{|l|}{ State at follow-up } \\
\hline Alive & 12 & 100 & 0 & - & & \\
\hline Dead & 0 & - & 12 & 100 & & \\
\hline
\end{tabular}

Table 2: miRNAs potentially over-expressed by resident BM cells from children with NB, as compared with BM resident cells from healthy children, ranked by fold change

\begin{tabular}{|c|c|c|c|c|}
\hline miRNA & Fold Change & $\begin{array}{c}\Delta \mathrm{Cq} \\
\text { Children with NB }\end{array}$ & $\begin{array}{c}\Delta \mathrm{Cq} \\
\text { Healthy children }\end{array}$ & $\Delta \Delta \mathbf{C q}$ \\
\hline hsa-miR-875-5p & 0.003 & 15.962 & 24.504 & 8.542 \\
\hline hsa-miR-221 & 0.008 & 6.103 & 13.110 & 7.007 \\
\hline hsa-miR-137 & 0.010 & 18.922 & 25.618 & 6.696 \\
\hline hsa-miR-29b & 0.010 & 10.864 & 17.503 & 6.639 \\
\hline hsa-miR-202 & 0.011 & 17.226 & 23.715 & 6.489 \\
\hline hsa-miR-17 & 0.012 & 1.854 & 8.232 & 6.378 \\
\hline hsa-let-7d & 0.015 & 5.870 & 11.955 & 6.084 \\
\hline hsa-miR-639 & 0.017 & 15.454 & 21.304 & 5.851 \\
\hline hsa-miR-320 & 0.017 & 5.863 & 11.700 & 5.837 \\
\hline hsa-miR-383 & 0.018 & 20.729 & 26.531 & 5.802 \\
\hline hsa-miR-617 & 0.019 & 21.831 & 27.524 & 5.693 \\
\hline hsa-miR-34a & 0.019 & 9.973 & 15.664 & 5.691 \\
\hline hsa-miR-376b & 0.019 & 22.075 & 27.761 & 5.686 \\
\hline hsa-miR-378 & $0 ., 021$ & 8.586 & 14.178 & 5.591 \\
\hline hsa-miR-599 & 0.023 & 18.730 & 24.170 & 5.440 \\
\hline hsa-miR-944 & 0.025 & 18.807 & 24.136 & 5.328 \\
\hline hsa-miR-10a & 0.026 & 10.927 & 16.204 & 5.277 \\
\hline hsa-miR-190b & 0.028 & 11.943 & 17.102 & 5.159 \\
\hline hsa-miR-369-3p & 0.031 & 18.744 & 23.760 & 5.016 \\
\hline
\end{tabular}

Genes potentially targeted by miR-221 and under-expressed by BM resident cells from children with NB

Since only miR-221 over-expression occurred in all patients with NB regardless of presence of metastatic cells in the BM as it is the case of altered erythrocyte maturation [17], we extracted the lists of genes potentially targeted by miR-221 from three different miRNA databases, Targetscan, miRanda and PicTar. In fact, TargetScan predicts biological targets of miRNAs by searching for the presence of conserved $8 \mathrm{mer}, 7 \mathrm{mer}$, and $6 \mathrm{mer}$ sites that 

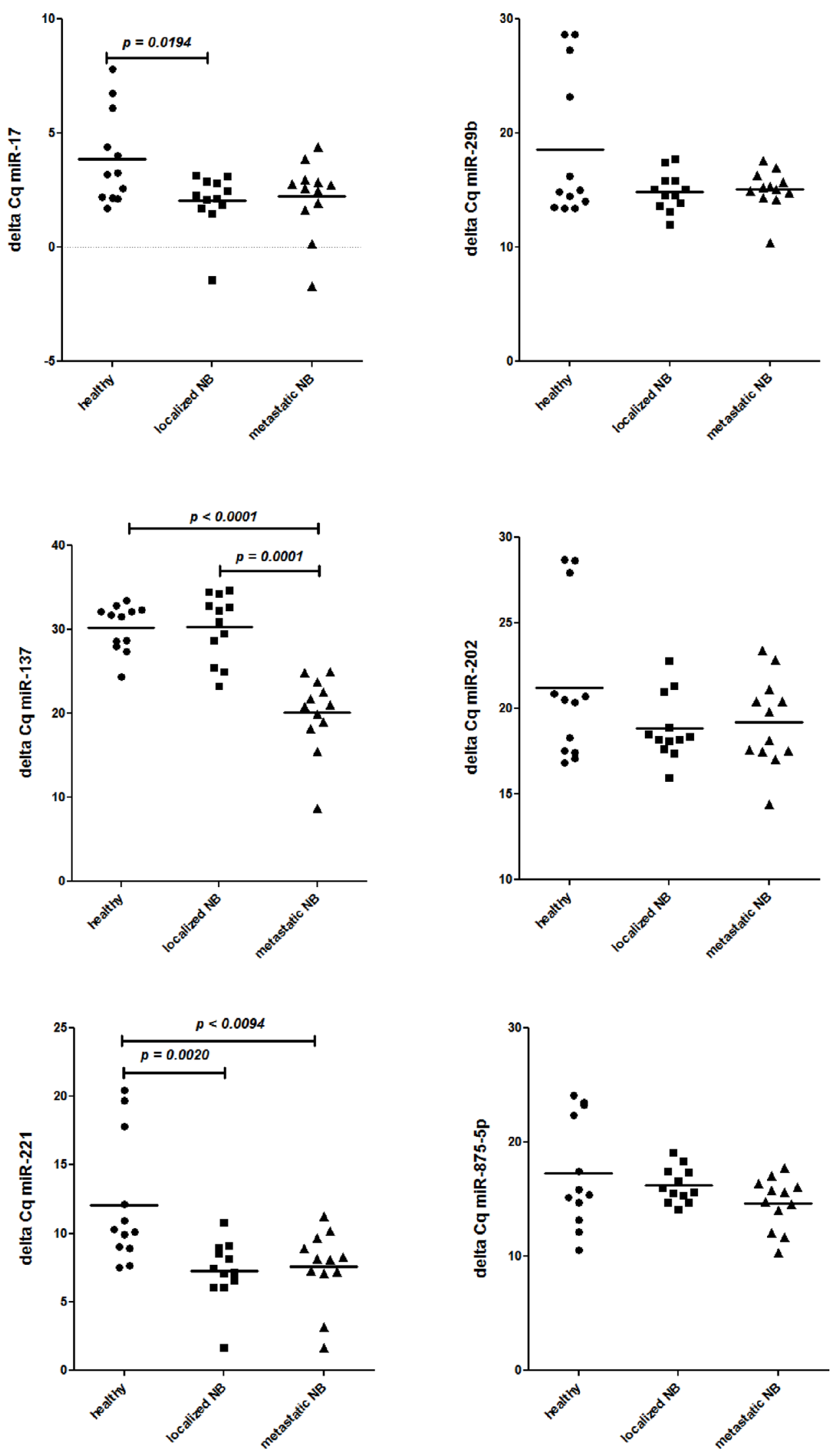

Figure 1: Expression levels (Delta Cq) of miR-17, miR-29b, miR-137, miR-202, miR-221, and miR-875-5p in BM resident cells from healthy children (closed circles) and from children with localized (closed squares) and metastatic (close triangles) NB. $N=12$ for each group. 
match the seed region of each miRNA [21] (http://www. targetscan.org); PicTar uses an algorithm to provide details, such as $3^{\prime}$ UTR alignments with predicted sites and links to various public databases [22] (http://pictar.mdc-berlin. $\mathrm{de}$ ), and miRanda prediction algorithm optimizes sequence complementarity using position-specific rules and relies on strict requirements of interspecies conservation [23] (http:// www.microrna.org/microrna/home.do).

By merging the three target gene lists from the three databases, the unique potential miR-221 target genes were extracted (Supplementary Table 5, left column), and then matched to the list of genes underexpressed by BM-resident cells from children with NB, as compared with healthy children (Supplementary Table 5 , right column). The latter list was derived from the GEO dataset GSE90689) [17] that were the most recent dataset available for this type of tissue.

The list of genes under-expressed by BM resident cells potentially targeted by miR-221, shown in Table 3, was then investigated for functional annotation (https://david. ncifcrf.gov/) [24] and pathway analysis (Ingenuity Systems
Pathway Analysis, www.qiagenbioinformatics.com). None of the genes shown in Table 3 were involved in heme and porphyrin biosynthesis, whereas two genes, namely BCL2L11 and BNIP3L, were related to mitochondria, where heme and porphyrin biosynthesis occurs.

\section{Expression of BCL2L11 and BNIP3L genes and their protein products by $B M$ resident cells from children with NB as compared with healthy children}

We thus evaluated BCL2L11 and BNIP $3 L$ gene expression levels in BM resident cells by RT-qPCR (Figure $3 \mathrm{~A}$ ) and their protein products by Western blot analysis (Figure 3B). BCL2L11 gene expression and protein levels in BM resident cells from children with NB and healthy children were not dissimilar. Conversely, $B N I P 3 L$ gene expression levels were confirmed to be significantly lower in BM resident cells from children with localized and metastatic NB, as compared with healthy children (Figure 3A). However, the level of BNIP3L

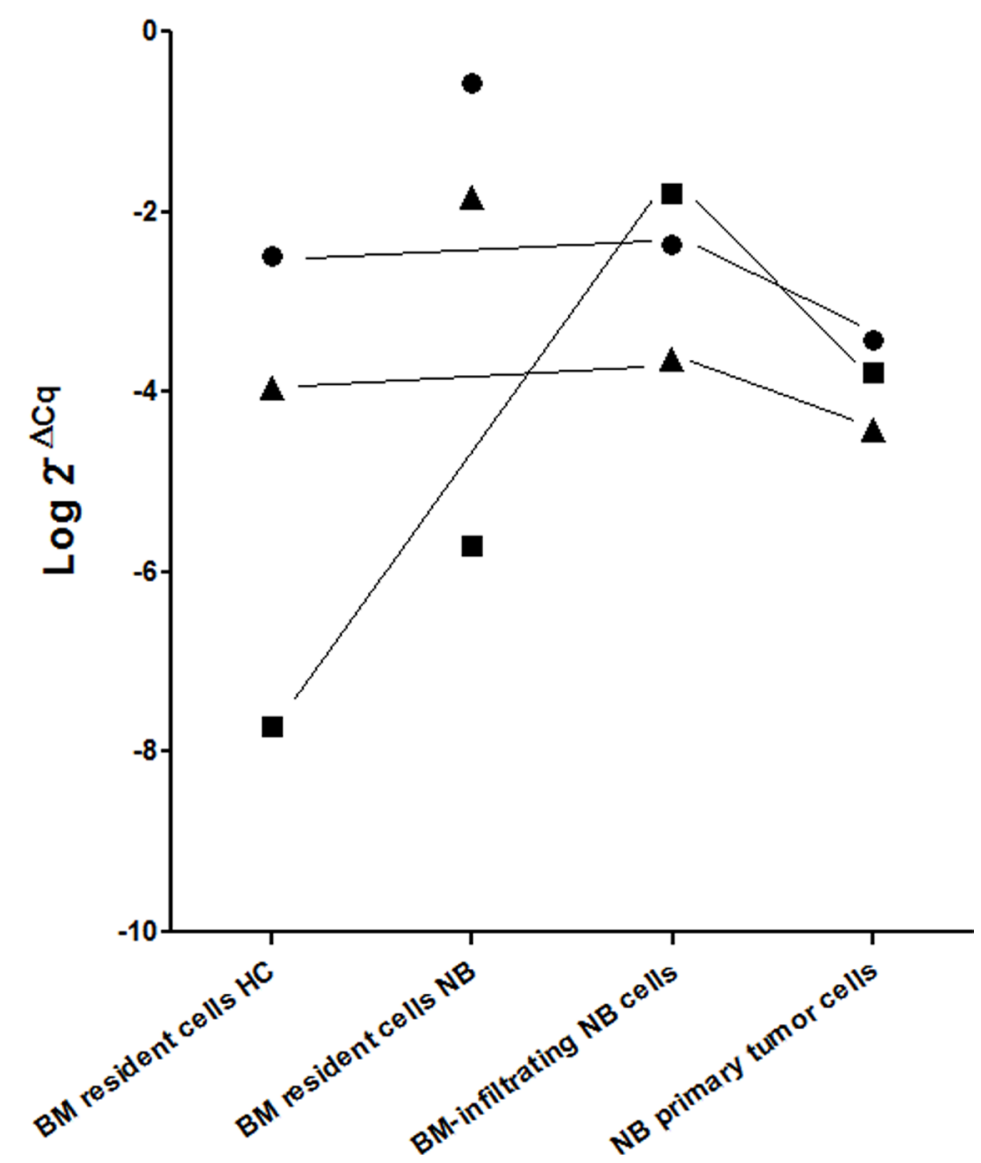

Figure 2: Mean expression levels $\left(\log 2^{-\Delta C q}\right)$ of miR-17 (closed circle), miR-137 (closed square) and miR-221 (closed triangle) in BM resident cells from healthy children (HC) and from children with NB, in BM-infiltrating NB cells and in NB primary tumors. MiR-17 and miR-221 expression levels in BM resident cells from healthy children were not significantly different from those of BM-infiltrating NB cells or primary tumor NB cells ( $p=n s$ ). MiR-137 expression levels in BM resident cells from healthy children were significantly lower than those of BM-infiltrating NB cells or primary tumor NB cells $(p=0.0004$ and $p=0.0002$, respectively). 
Table 3: Genes potentially targeted by miR-221 and under-expressed in BM resident cells from children with NB, as compared with healthy children

\section{Genes}

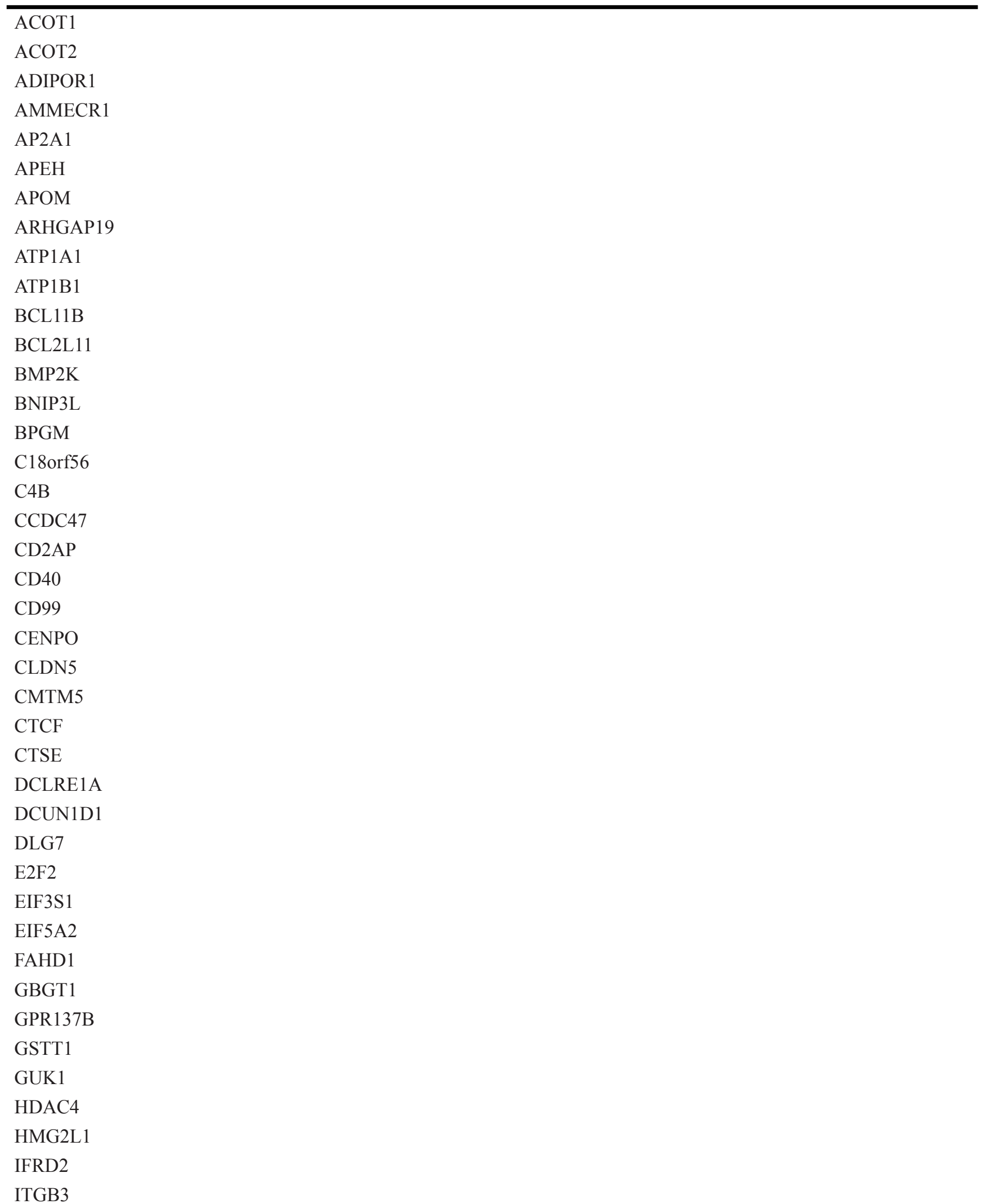

ITGB3 
LHFPL2

MARCH8

MCM5

MST1

MYBL2

NAP1L5

NAPA

NGRN

NUTF2

PAIP1

PES1

PFDN6

PIGC

PLEKHF1

PPP1R8

PRPS1

PSMB5

QARS

SIGLECP3

SLC25A15

SLC25A37

SLC25A39

TIGD6

TIMP3

TMEM85

TMPRSS9

WDR40A

WDR89

WHSC1

WRN

WSB2

YWHAG

ZNF557

protein product, Nix, was heterogeneous both in children with NB and healthy children (Figure 3B), without any significant reduction in children with NB.

\section{DISCUSSION}

The transfer of miRNAs, key regulators of gene expression, is considered as one of the major causes leading to modification of physiological functions occurring in tissues distant from the primary tumor [2-4]. Therefore, we have investigated whether the selective erythrocyte impairment observed in children with NB, regardless of the presence of metastatic cells in the BM microenvironment [17], was mediated by a specific miRNA secreted by NB cells.

Our results did not support this hypothesis. First of all, the miRNA expression profile of BM resident cells from children with NB was not significantly different from that of healthy children. By considering the fold change we identified six miRNAs potentially over-expressed in NB by more than 150 fold, as compared with healthy children. Validation experiments performed in a larger number of samples from each group confirmed the differential expression for $50 \%$ of these miRNAs. The low percentage of validated miRNAs may be depended on the high heterogeneity of NB [6] or on the different platform 
used for the test and validation experiments. This finding was in line with other studies on miRNA expression in NB tumors $[9,10,12-14,20]$.

Three miRNAs, namely miR-17, miR-137 and miR-221, were over-expressed by BM resident cells from children with NB, as compared with healthy children. However, miR-17 was over-expressed only in children with localized NB and miR-137 only in children with metastatic NB. Whereas high level of miR-137 were likely due to close contact between BM-infiltrating NB cells and $\mathrm{BM}$ resident cells, the increased expression of miR-17 and
miR-221 was unlikely to be a consequence of a transfer from NB primary tumor cells, because the levels in NB cells were similar to that of BM resident cells from healthy children.

Since the altered erythrocyte maturation, recently documented in children with NB [17], occurred in both localized and metastatic NB, we focused our attention on miR-221 that was significantly over-expressed by BM resident cells from all children with NB. All the genes potentially targeted by miR-221 were extracted from three public miRNA databases and compared to the full list of

A
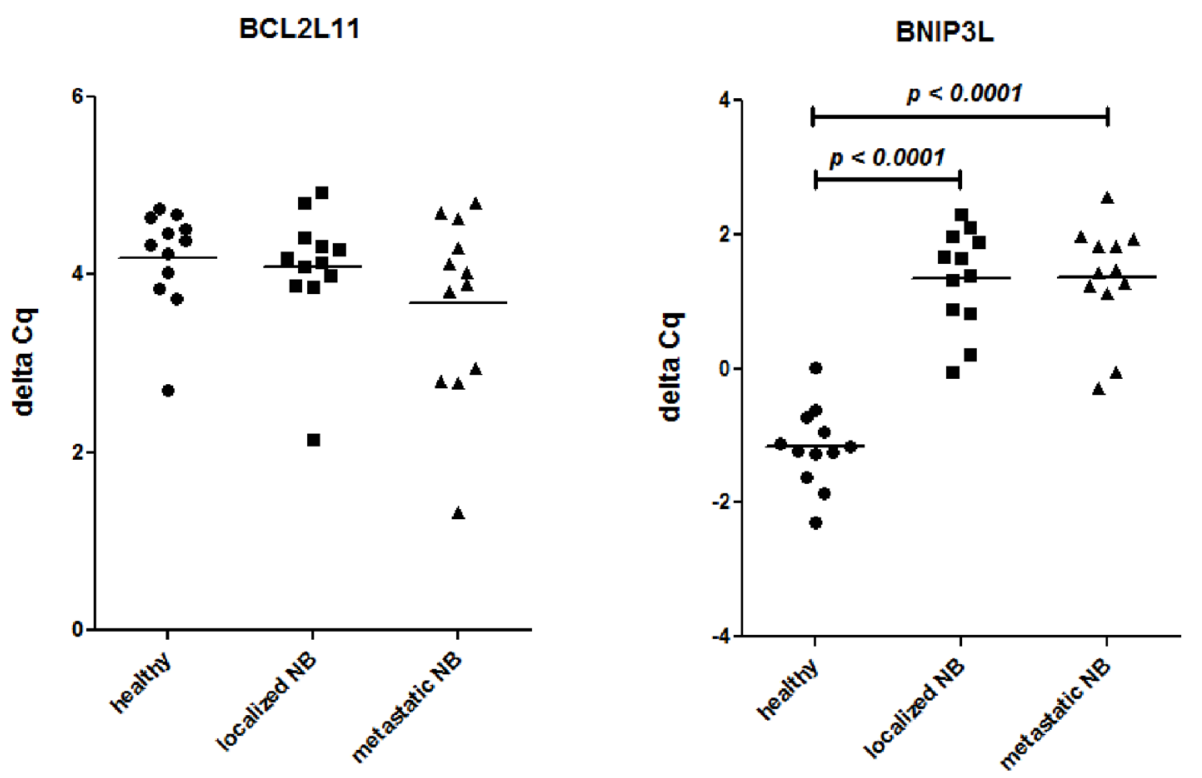

B

$\mathrm{NB} \# 1 \quad \mathrm{NB} \# 2 \quad \mathrm{NB} \# 3 \quad \mathrm{NB} \# 4$
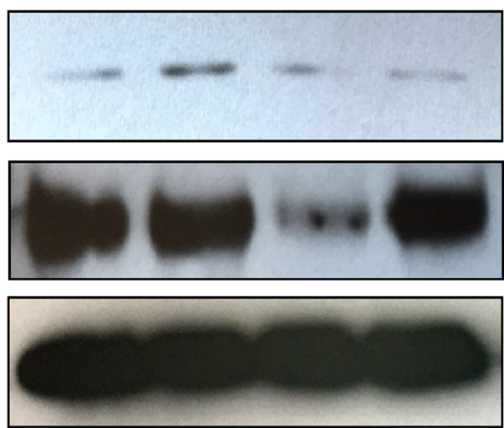

HD\#1 HD\#2 HD\#3 HD\#4

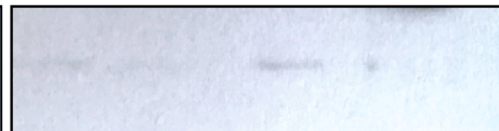

BCL2L11/Bim

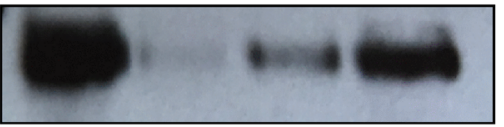

BNIP3L/Nix

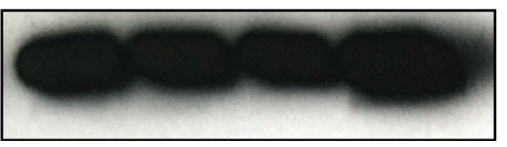

GAPDH

Figure 3: (A) Expression levels (Delta Cq) of BCL2L11 and BNIP3L mRNA in BM resident cells from healthy children (closed circles) and from children with localized (closed squares) and metastatic (close triangles) NB. $N=12$ for each group. (B) Western blot analysis of BCL2L11 and BNIP3L protein products, Bim and Nix, respectively, in BM resident cells from four children with NB (NB\#1 to \#4) and from four healthy children (HD\#1 to \#4). 
genes that were under-expressed in BM resident cells from children with NB, as compared with healthy children (GSE90689) [17]. The GSE90689 was the most recent dataset containing gene expression data from a total of 57 $\mathrm{BM}$ resident cells from both healthy children and children with NB. The older dataset, GSE25624 [19], contained data from a total of 40 samples. Recently, a dataset containing expression data of BM resident cells from children with NB in comparison with expression data of BM-infiltrating NB cells (GSE 94035) [25] became available, but the lack of expression data of BM resident cells from healthy children made it unsuitable to answer our question.

The genes under-expressed by BM resident cells from children with NB, as compared with healthy children, potentially targeted by miR-221 did not annotate in the pathway of heme and porphyrin synthesis. Two genes, BCL2L11 and BNIP3L, expressed in mitochondria where heme and porphyrin synthesis occurs, were tested for gene and protein expression, but the results did not support a role for them in the impairment of late erythropoiesis. MiR-221 has been implicated in the regulation of early erythropoiesis [26], since its over-expression following lentiviral transfection in $\mathrm{CD} 34^{+}$precursors reduced the levels of its target gene CKIT and impaired stem cell activity. In our dataset, no difference in CKIT expression levels was found, confirming that early erythropoiesis was not impaired in children with NB.

In conclusion, our data did not support the hypothesis that selective erythrocyte maturation may be mediated by miRNAs released by primary NB tumor cells nor by miR-221. However, we cannot rule out the possibility that other miRNAs, not evaluated in the present study, may be involved in the process. Thus, we have made public (see Supplemental Tables) miRNA expression data of BM resident cells from children with NB and from healthy children described here, as well as miRNA expression data of NB primary tumors and BM-infiltrating NB cells that were published previously [20].

\section{MATERIALS AND METHODS}

\section{Patients and controls}

Patients included in the study were consecutively diagnosed with NB between January 2007 and December 2008. The main features of the patients are summarized in Table 1. Demographic, genetic, clinical and follow-up data were retrieved from the Italian NB Registry [27]. After diagnosis, patients were treated according to the European protocol suited for her/his risk category, dependent on age, stage and MYCN status [5].

As controls we used BM samples obtained from healthy siblings of leukemia patients admitted at the Gaslini Institute to undergo BM transplants.

Written consent for research use of samples and clinical data was obtained by the legal guardians. The study was approved by the Gaslini Institute Ethical Committee and all analyses were performed according to the Helsinki declaration.

\section{BM resident cells from NB patients and healthy children}

BM resident cells were immune-selected from BM samples taken at diagnosis from 24 children with NB and from 12 healthy children, as previously described [19]. The procedure was applied to all BM samples to rule out the possibility that the procedure per se could influence the results. From each group of samples, i.e., from localized or metastatic NB and healthy children, 4 samples were randomly selected to perform complete miRNA profiling. All twelve samples of each group were used in the validation experiments.

\section{RNA extraction, miRNA profiling and validation}

Total RNA and miRNA fractions were extracted from immune-selected BM resident cells using the miRNeasyMini kit (Qiagen, Hilden, Germany) according to manufacturer's protocols. Quality of the RNA fractions was evaluated using the BioAnalyzer 2100 (Agilent Technologies, Santa Clara, CA, USA).

Forty ng of the miRNA fraction were reverse transcribed using the Megaplex RT Primers Human Pool A and B (Thermo Fisher, Milan, Italy). At the end of the reaction, each RT product was amplified with the Megaplex PreAmp Primers A and B for 25 cycles. Then, the amplification products of 4 samples from each group were loaded onto MicroRNA TaqMan Card A and $\mathrm{B}$, respectively. Card amplifications were performed on ViiA7 equipment for 40 cycles (Thermo Fisher). Normalization of expression was made by using the mean expression value of U6 small RNA measured in each card [20].

Validation was performed by loading the amplification products in a 96 well plate in duplicates with the specific TaqMan ${ }^{\odot}$ human microRNA assays (Thermo Fisher: hsa-miR-17, catalog \#002308; hsa-miR-137, catalog \#01129; hsa-miR-202, catalog \#002363; hsa-miR-221, catalog \#000524; hsa-miR-29b, catalog \#000413; hsa-miR875-5p, catalog \#002203; U6 snRNA, catalog \#001973). Results were expressed as delta $\mathrm{Cq}$ by subtracting the $\mathrm{Cq}$ value obtained for U6 small RNA from the Cq value of each miRNA [28].

\section{Genes targeted by miR-221}

The lists of genes potentially targeted by miR221 were downloaded from three public miRNA databases:Targetscan(http://www.targetscan.org/vert_71/), miRANDA(http://www.microrna.org/microrna/home.do) and PicTar (http://pictar.mdc-berlin.de/). The three lists 
were then merged to obtain a comprehensive list of all the genes potentially targeted by miR-221. The unique gene list was then compared with the list of genes significantly under-expressed by BM resident cells from children with NB, as compared with healthy subjects [19], deposited in National Center for Biotechnology Information Gene Expression Omnibus (GEO, http://www.ncbi.nlm.nih.gov/ geo/, accession GSE90689).

\section{Gene expression analysis of potential miRNA targets}

One-hundred ng of total RNA from the 36 samples under study were reverse transcribed as described [19, 29], and then amplified in triplicate with the specific TaqMan ${ }^{\odot}$ human gene expression assays for $B C L 2 L 11, B N I P 3 L$ and GAPDH (Thermo Fisher, catalog\# Hs00708019_s1, Hs00188949_m1 and 4333764F, respectively). Results were expressed as delta $\mathrm{Cq}$ by subtracting the $\mathrm{Cq}$ value obtained for GAPDH from the Cq value of each target gene [28].

\section{Western blot}

Protein lysates of BM resident cells from four children with metastatic NB and four healthy children, all included in Table 1, were obtained using Cell Extraction Buffer (Thermo Fisher), following manufacturer's protocol. Protein concentration was assessed using a BCA assay (Bio-Rad Laboratories, Segrate, Italy) and absorbance at $562 \mathrm{~nm}$ was measured using Infinite ${ }^{\circledR} 200$ PRO spectrometer (Tecan Italia Srl, Cernusco Sul Naviglio, Italy). Protein lysates (40 $\mu \mathrm{g}$ per lane) were resolved on SDS $10 \%$ polyacrylamide gels and were transferred to nitrocellulose membranes. The membranes were sequentially incubated with antiBim/BCL2L11 (mouse monoclonal, H-5) and anti-Nix/ BNIP3L (mouse monoclonal, H-8), both from Santa Cruz Biotechnology Inc., Heidelberg, Germany, and with antiGAPDH (rabbit monoclonal antibody from Cell Signaling Europe, Leiden, The Netherlands).

All primary antibodies were diluted at 1:200 in TBS $0.1 \%$ Tween (Sigma Aldrich, Milano, Italy) and 5\% nonfat dry milk (BioRad), and membranes were incubated overnight at $4^{\circ} \mathrm{C}$ in constant agitation. Secondary reagents (HRP-conjugated horse anti-mouse or goat antirabbit antibodies (Cell signaling) were diluted following manufacturer's protocol and membranes were incubated for $1 \mathrm{~h}$ at RT in constant agitation. Immune complexes were visualized using Clarity ECL Western Blot Substrate (BioRad) according to the manufacturer's instructions.

\section{Statistical analysis}

Analysis of miRNA Cq values from high-throughput qPCR assays was conducted using the HTqPCR package [30] of Bioconductor [31], which runs on $\mathrm{R}$ statistical computing environment (http://www.R-project.org/).
Difference in miRNA expression levels in BM resident cells from the three groups of samples were tested by performing Mann-Whitney rank test. Adjusting $p$ values to face multiple comparison problems were performed according to the Benjamini-Hochberg procedure [32].

Differences in miRNA and gene expression levels evaluated by RT-qPCR in 96-well plates in all 36 samples were tested by the Mann-Whitney rank test using the Prism software (GraphPad Software Inc., La Jolla, CA, USA).

\section{Author contributions}

S.S. performed miRNA and gene expression experiments and interpreted the data; F.M. performed immune-selection of $\mathrm{BM}$ resident cells and western blot experiments and interpreted the data; L.P. and C.L. performed statistical analysis; G.E. provided clinical data from the Italian NB Registry; P.S. contributed to the study design, interpreted the data and critically revised the manuscript; M.V.C. designed the study, analyzed and interpreted the data and wrote the manuscript.

All authors read and approved the final manuscript.

\section{ACKNOWLEDGMENTS}

FM was recipient of a fellowship from Fondazione Veronesi and GE is recipient of a fellowship from Fondazione Italiana per la Lotta al Neuroblastoma. We thank Barbara Carlini for excellent technical assistance.

\section{CONFLICTS OF INTEREST}

The authors declare no conflicts of interest.

\section{FUNDING}

This work was supported by Fondazione Italiana per la Lotta al Neuroblastoma (Progetto clinico to M.V.C)

\section{REFERENCES}

1. Valinezhad Orang A, Safaralizadeh R, KazemzadehBavili M. Mechanisms of miRNA-Mediated Gene Regulation from Common Downregulation to mRNASpecific Upregulation. Int J Genom. 2014; 2014:970607.

2. Frediani JN, Fabbri M. Essential role of miRNAs in orchestrating the biology of the tumor microenvironment. Mol Cancer. 2016; 15:42.

3. Gopal SK, Greening DW, Rai A, Chen M, Xu R, Shafiq A, Mathias RA, Zhu HJ, Simpson RJ. Extracellular vesicles: their role in cancer biology and epithelial-mesenchymal transition. Biochem J. 2017; 474:21-45.

4. Undi RB, Kandi R, Gutti RK. MicroRNAs as Haematopoiesis Regulators. Adv Hemat. 2013; 2013:695754. 
5. Cohn SL, Pearson AD, London WB, Monclair T, Ambros PF, Brodeur GM, Faldum A, Hero B, Iehara T, Machin D, Mosseri V, Simon T, Garaventa A, et al. The International Neuroblastoma Risk Group (INRG) classification system: an INRG Task Force report. J Clin Oncol. 2009; 27:289-297.

6. Matthay KK, Maris JM, Schleiermacher G, Nakagawara A, Mackall CL, Diller L, Weiss WA. Neuroblastoma. Nat Rev Dis Primers. 2016; 2:16078.

7. Stallings RL. MicroRNA involvement in the pathogenesis of neuroblastoma: potential for microRNA mediated therapeutics. Curr Pharm Des. 2009; 15:456-462.

8. Althoff K, Schulte JH, Schramm A. Towards diagnostic application of non-coding RNAs in neuroblastoma. Exp Rev Mol Diagn. 2016; 16:1307-1313.

9. Guo J, Dong Q, Fang Z, Chen X, Lu H, Wang K, Yin Y, Cai X, Zhao N, Chen J, Zen K, Zhang J, Zhang CY. Identification of miRNAs that are associated with tumor metastasis in neuroblastoma. Cancer Biol Ther. 2010; 9:446-452.

10. Terrile M, Bryan K, Vaughan L, Hallsworth A, Webber H, Chesler L, Stallings RL. miRNA expression profiling of the murine TH-MYCN neuroblastoma model reveals similarities with human tumors and identifies novel candidate miRNAs. PLoS One. 2011; 6:e28356.

11. Schulte JH, Schowe B, Mestdagh P, Kaderali L, Kalaghatgi P, Schlierf S, Vermeulen J, Brockmeyer B, Pajtler K, Thor T, de Preter K, Speleman F, Morik K, et al. Accurate prediction of neuroblastoma outcome based on miRNA expression profiles. Int J Cancer. 2010; 127:2374-2385.

12. Schulte JH, Marschall T, Martin M, Rosenstiel $P$, Mestdagh P, Schlierf S, Thor T, Vandesompele J, Eggert A, Schreiber S, Rahmann S, Schramm A. Deep sequencing reveals differential expression of microRNAs in favorable versus unfavorable neuroblastoma. Nucleic Acids Res. 2010; 38:5919-5928.

13. De Preter K, Mestdagh P, Vermeulen J, Zeka F, Naranjo A, Bray I, Castel V, Chen C, Drozynska E, Eggert A, Hogarty MD, Izycka-Swieszewska E, London WB, et al. miRNA expression profiling enables risk stratification in archived and fresh neuroblastoma tumor samples. Clin Cancer Res. 2011; 17:7684-7692.

14. De Mariano M, Stigliani S, Moretti S, Parodi F, Croce M, Bernardi C, Pagano A, Tonini GP, Ferrini S, Longo L. A genome-wide microRNA profiling indicates miR-424-5p and $\mathrm{miR}-503-5 \mathrm{p}$ as regulators of ALK expression in neuroblastoma. Oncotarget. 2017; 8:56518-56532. https:// doi.org/10.18632/oncotarget.17033.

15. Mestdagh P, Bostrom AK, Impens F, Fredlund E, Van Peer G, De Antonellis P, von Stedingk K, Ghesquiere B, Schulte S, Dews M, Thomas-Tikhonenko A, Schulte JH, Zollo $\mathrm{M}$, et al. The miR-17-92 microRNA cluster regulates multiple components of the TGF-beta pathway in neuroblastoma. Mol Cell. 2010; 40:762-773.
16. Scaruffi P, Stigliani S, Moretti S, Coco S, De Vecchi C, Valdora F, Garaventa A, Bonassi S, Tonini GP. TranscribedUltra Conserved Region expression is associated with outcome in high-risk neuroblastoma. BMC Cancer. 2009; 9:441.

17. Morandi F, Barco S, Stigliani S, Croce M, Persico L, Lagazio C, Scuderi F, Belli ML, Montera M, Cangemi G, Pozzi S, Rigo V, Scaruffi P, et al. Altered erythropoiesis and decreased number of erythrocytes in children with neuroblastoma. Oncotarget. 2017; 8:53194-53209. https:// doi.org/10.18632/oncotarget.18285.

18. Mestdagh P, Feys T, Bernard N, Guenther S, Chen C, Speleman F, Vandesompele J. High-throughput stemloop RT-qPCR miRNA expression profiling using minute amounts of input RNA. Nucleic Acids Res. 2008; 36:e143.

19. Scaruffi P, Morandi F, Gallo F, Stigliani S, Parodi S, Moretti S, Bonassi S, Fardin P, Garaventa A, Zanazzo G, Pistoia V, Tonini GP, Corrias MV. Bone marrow of neuroblastoma patients shows downregulation of CXCL12 expression and presence of IFN signature. Pediatr Blood Cancer. 2012; 59:44-51.

20. Stigliani S, Scaruffi P, Lagazio C, Persico L, Carlini B, Varesio L, Morandi F, Morini M, Gigliotti AR, Esposito MR, Viscardi E, Cecinati V, Conte M, Corrias MV. Deregulation of focal adhesion pathway mediated by miR$659-3 p$ is implicated in bone marrow infiltration of stage $M$ neuroblastoma patients. Oncotarget. 2015; 6:13295-13308. https://doi.org/10.18632/oncotarget.3745.

21. Lewis BP, Burge CB, Bartel DP. Conserved seed pairing, often flanked by adenosines, indicates that thousands of human genes are microRNA targets. Cell. 2005; 120:15-20.

22. Krek A, Grun D, Poy MN, Wolf R, Rosenberg L, Epstein EJ, MacMenamin P, da Piedade I, Gunsalus KC, Stoffel M, Rajewsky N. Combinatorial microRNA target predictions. Nat Genet. 2005; 37:495-500.

23. John B, Enright AJ, Aravin A, Tuschl T, Sander C, Marks DS. Human MicroRNA targets. PLoS biology. 2004; 2:e363.

24. Huang DW, Sherman BT, Tan Q, Kir J, Liu D, Bryant D, Guo Y, Stephens R, Baseler MW, Lane HC, Lempicki RA. DAVID Bioinformatics Resources: expanded annotation database and novel algorithms to better extract biology from large gene lists. Nucleic Acids Res. 2007; 35:W169-175.

25. Rifatbegovic F, Frech C, Abbasi MR, Taschner-Mandl S, Weiss T, Schmidt WM, Schmidt I, Ladenstein R, Ambros IM, Ambros PF. Neuroblastoma cells undergo transcriptomic alterations upon dissemination into the bone marrow and subsequent tumor progression. Int J Cancer. 2017; 142:297-307.

26. Felli N, Fontana L, Pelosi E, Botta R, Bonci D, Facchiano F, Liuzzi F, Lulli V, Morsilli O, Santoro S, Valtieri M, Calin GA, Liu CG, et al. MicroRNAs 221 and 222 inhibit normal erythropoiesis and erythroleukemic cell growth via kit receptor down-modulation. Proc Natl Acad Sci USA. 2005; 102:18081-18086. 
27. Haupt R, Garaventa A, Gambini C, Parodi S, Cangemi G, Casale F, Viscardi E, Bianchi M, Prete A, Jenkner A, Luksch R, Di Cataldo A, Favre C, et al. Improved survival of children with neuroblastoma between 1979 and 2005: a report of the Italian Neuroblastoma Registry. J Clin Oncol. 2010; 28:2331-2338.

28. Livak KJ, Schmittgen TD. Analysis of relative gene expression data using real-time quantitative PCR and the 2(-Delta Delta C(T)) Method. Methods. 2001; 25:402-408.

29. Corrias MV, Haupt R, Carlini B, Cappelli E, Giardino S, Tripodi G, Tonini GP, Garaventa A, Pistoia V, Pistorio A. Multiple target molecular monitoring of bone marrow and peripheral blood samples from patients with localized neuroblastoma and healthy donors. Pediatr Blood Cancer. 2012; 58:43-49.

30. Dvinge $\mathrm{H}$, Bertone P. HTqPCR: high-throughput analysis and visualization of quantitative real-time PCR data in R. Bioinformatics. 2009; 25:3325-3326.

31. Gentleman RC, Carey VJ, Bates DM, Bolstad B, Dettling M, Dudoit S, Ellis B, Gautier L, Ge YC, Gentry J, Hornik K, Hothorn T, Huber W, et al. Bioconductor: open software development for computational biology and bioinformatics. Genome Biol. 2004; 5.

32. Benjamini Y, Hochberg Y. Controlling the False Discovery Rate - a Practical and Powerful Approach to Multiple Testing. J Roy Stat Soc B Met. 1995; 57:289-300. 